\title{
Holographic filters
}

\section{Hung-Te Hsieh, Zhenyu Li, Demetri Psaltis}

Hung-Te Hsieh, Zhenyu Li, Demetri Psaltis, "Holographic filters," Proc. SPIE 5521, Organic Holographic Materials and Applications II, (26 October 2004); doi: $10.1117 / 12.565189$

SPIE Event: Optical Science and Technology, the SPIE 49th Annual Meeting, 2004, Denver, Colorado, United States 


\title{
Holographic filters
}

\author{
Hung-Te Hsieh, Zhenyu Li, Demetri Psaltis \\ Department of Electrical Engineering, California Institute of Technology \\ Pasadena, CA USA 91125
}

\begin{abstract}
We present two applications of volume holographic filters in the reflection geometry. A passive athermal holographic filter design is realized through the mutual compensation between the temperature coefficients of the bulk hologram and a variable incident angle controlled by a bimetallic cantilever. Seven holograms are multiplexed to constitute a multiline filter which can be used to emulate specific absorption or emission spectra for spectroscopic applications.
\end{abstract}

Keywords: Holographic filters, wavelength-division multiplexing (WDM), angle multiplexing, spectroscopy

\section{VOLUME HOLOGRAPHIC GRATINGS AND THEIR FILTERING PROPERTIES}

A volume holographic grating (VHG) consists of a modulated refractive index pattern imprinted inside a bulk recording material (e.g., photorefractive crystals ${ }^{1}$, photosensitive polymers ${ }^{2}$ ).

$$
n(z)=n+\Delta n \cos \left(\frac{2 \pi}{\Lambda} z\right)
$$

where $\Lambda$ is the period of the grating which is oriented along the $\mathrm{z}$ coordinate. Once written, the grating can act as an efficient coupler between plane waves. The coupling property is largely specified by two important parameters. The first is the grating period $\Lambda$, which determines the Bragg condition and therefore the appropriate interacting optical wavelength $\lambda$. As shown in Fig. 1, if the wavevectors of two optical fields and the grating vector $K$ constitute the sides of a triangle, the optical fields can be coupled through the holographic grating because the conservation of momentum is satisfied. The relevant Bragg condition in this case is
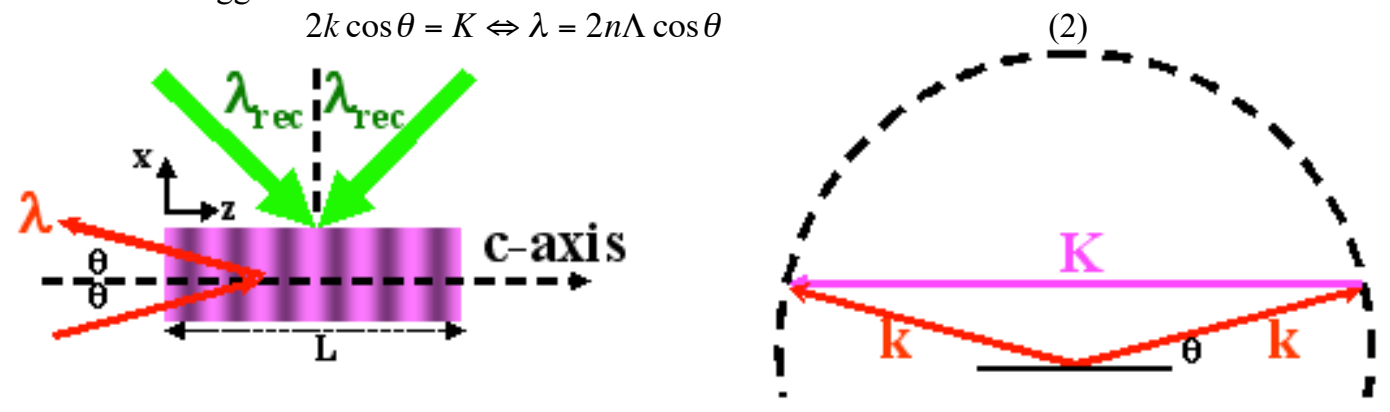

Figure 1. Recording a holographic grating inside a $\mathrm{LiNbO}_{3}$ crystal at $\lambda_{\text {rec }}=488 \mathrm{~nm}$ in the transmission geometry and then operating it as a filter in the reflection geometry.

where $n$ is the refractive index of $\lambda$ inside the material and $\theta$ is the angle of incidence. Wavelength components which meet the Bragg condition will be diffracted by the grating and those that do not will be transmitted instead. Therefore the holographic grating acts as a filter, discriminating between the Bragg wavelength and the rest of the spectrum.

The strength of coupling between the optical fields is in turn decided by the refractive index modulation $\Delta n$. The diffraction efficiency of interest $\eta$ is defined as the intensity ratio of the reflected and incident optical fields at the front surface; it has been shown to $\mathrm{be}^{3}$

$$
\eta=\frac{\kappa^{2} \sinh ^{2} s L}{s^{2} \cosh ^{2} s L+(\Delta \beta)^{2} \sinh ^{2} s L}
$$

where $\kappa=\pi \Delta n / \lambda \cos \theta$ is the coupling constant, $L$ is the length of the grating, $\Delta \beta=k \cos \theta-K / 2$ determines the detuning factor due to an angular or wavelength mismatch and $s^{2}=\kappa^{2}-(\Delta \beta)^{2}$. The stopband of such a filter is marked by the 
condition $\kappa \geq|\Delta \beta|$. Within the stopband, the reflected optical field grows exponentially towards the front surface at the expense of a corresponding exponential decay of the incident field towards the end surface.

In the experiments we describe in this paper, holographic filters are recorded in an iron-doped lithium niobate crystal $\left(\mathrm{LiNbO}_{3}: \mathrm{Fe}, 0.05\right.$ wt. $\% \mathrm{Fe}_{2} \mathrm{O}_{3}$ ) by interfering two coherent continuous wave (cw) laser beams at $488 \mathrm{~nm}$ inside the crystal, as shown in Fig. 1. The dimensions of the sample are $2.0 \mathrm{~cm} \times 1.0 \mathrm{~cm} \times 0.3 \mathrm{~cm}$ with its c-axis running parallel to the longest edge and the concentration of $\mathrm{Fe}^{2+}$ is $1.28 \times 10^{24} \mathrm{~m}^{-3}$. By properly choosing the angle between the recording beams we can control the Bragg wavelength of the grating operated in the reflection geometry.

\section{ATHERMAL HOLOGRAPHIC FILTERS}

A temperature-independent spectral filter is highly desirable for most applications and particularly for telecommunications. Athermal filter designs have been carried out in arrayed-waveguide gratings ${ }^{4}$ (AWG) and fiber Bragg gratings ${ }^{5}$ (FBG). Here we review the design and experimental demonstration of an athermal holographic filter utilizing a passive thermally actuated microelectromechanical system (MEMS) mirror, which compensates for the drift of the Bragg wavelength when the temperature changes.

The temperature dependence of the Bragg wavelength of a holographic filter is mainly a result of two mechanisms. The first is the thermal expansion and contraction of the bulk material; the second is the thermal dependence of the refractive index. When the temperature changes by $\Delta T$, the Bragg wavelength of the filter will have a corresponding shift of $\Delta \lambda$. If we alter the incident angle by $\Delta \theta$ in such a way that the Bragg wavelength remains constant in spite of the temperature drift, we will have

$$
\lambda=2 n(1+a \Delta T) \Lambda(1+b \Delta T) \cos (\theta+\Delta \theta)
$$

where a linear temperature dependence on both mechanisms has been assumed. Combine Eq. (2) and (4), we end up with an explicit relation between $\Delta \theta$ and $\Delta T$ for a constant Bragg wavelength:

$$
\frac{\cos (\theta+\Delta \theta)}{\cos \theta}=\frac{1}{(1+a \Delta T)(1+b \Delta T)}
$$

As both coefficients $a$ and $b$ are positive ${ }^{6,7}$, we know that when the temperature increases, the Bragg wavelength will shift to a longer wavelength. Eq. (5) tells us that in order to compensate for such a shift, we need to increase the incident angle properly, i.e., to tilt the incident beam away from the normal. Conversely, to undo the effect brought about by a decrease in temperature, we have to adjust the beam towards the normal.

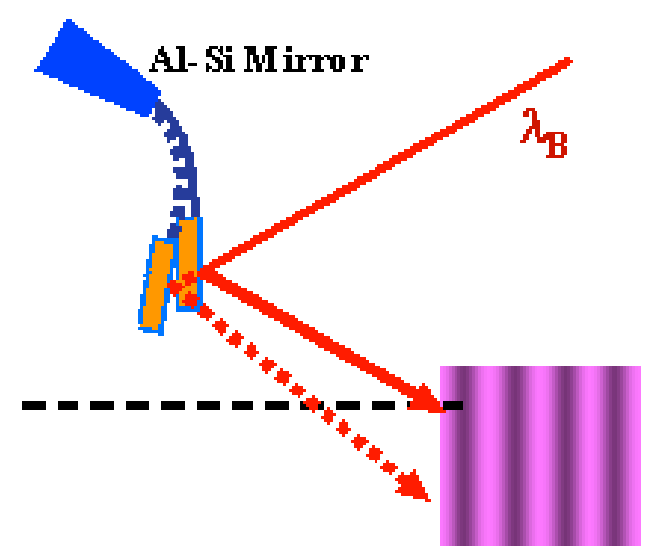

(a)

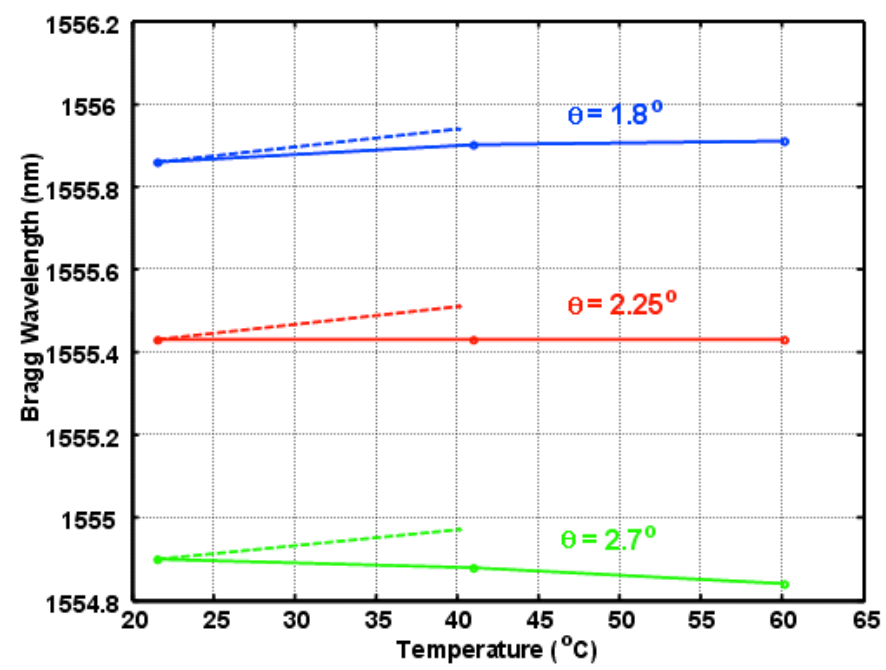

(b)

Figure 2. (a) The athermal design of a holographic filter utilizing an Al-Si composite beam microactuator whose tip deflects as the temperature changes. (b) The Bragg wavelengths measured with the athermal design for three different incident angles versus temperature.

We propose an athermal holographic filter design in Fig. 2(a) based on Eq. (5). The principle of operation is described as follows: the bimetallic composite beam is composed of two properly chosen materials (in this case, aluminum and 
silicon) whose thermal expansion coefficients (TECs) are different; as temperatures changes, the composite beam deflects such that the incident beam reflected off it hits the holographic filter at a slightly different angle and the Bragg wavelength is maintained constant despite the thermal fluctuation. We have to operate the filter away from normal incidence in order to accommodate a negative temperature drift as well as to avoid the nonlinearity around $\theta=0^{\circ}$ $(\Delta \theta(\Delta T)$ is a highly nonlinear function near normal incidence).

The transmittance of a holographic filter at an incident angle $\theta=2.25^{\circ}$ is shown in Fig. 3(a) for three different temperatures. The Bragg wavelength $\lambda$ of the filter at $21.79^{\circ} \mathrm{C}$ is measured to be $1556.61 \mathrm{~nm}$. The filter efficiency is dropping due to hologram decay at elevated temperatures since the hologram was not thermally fixed ${ }^{8}$. The Bragg wavelength drifts about $0.16 \mathrm{~nm}(20 \mathrm{GHz})$ over a temperature range of $37^{\circ} \mathrm{C}$. With the counteracting MEMS mirror in action, the measured filter transmittance is plotted in Fig. 3(b). Compared with Fig. 3(a), the drift of the Bragg wavelength is indeed compensated for by the deflection of the mirror.

In Fig. 2(b) we plot the Bragg wavelengths measured with the athermal design for three different incident angles. The dashed lines indicate the drifts of Bragg wavelengths due to the raised temperature in the absence of mirror compensation. We can see that at $\theta=2.25^{\circ}$, the Bragg wavelength remains constant between $21^{\circ} \mathrm{C}$ and $60^{\circ} \mathrm{C}$, which corroborates the validity of our athermal design. The Bragg wavelengths for $\theta=1.8^{\circ}$ are seen to increase with the rising temperature despite the counteracting MEMS operation although they do not increase quite as much as when there's no compensation mechanism; on the other hand, the Bragg wavelengths for $\theta=2.7^{\circ}$ are decreasing with the rising temperature obviously because they are overcompensated by the MEMS mirror deflection. The compensation can be done easily in practice because the response time of the MEMS mirror is much shorter than that of the bulky $\mathrm{LiNbO}_{3}$ crystal.

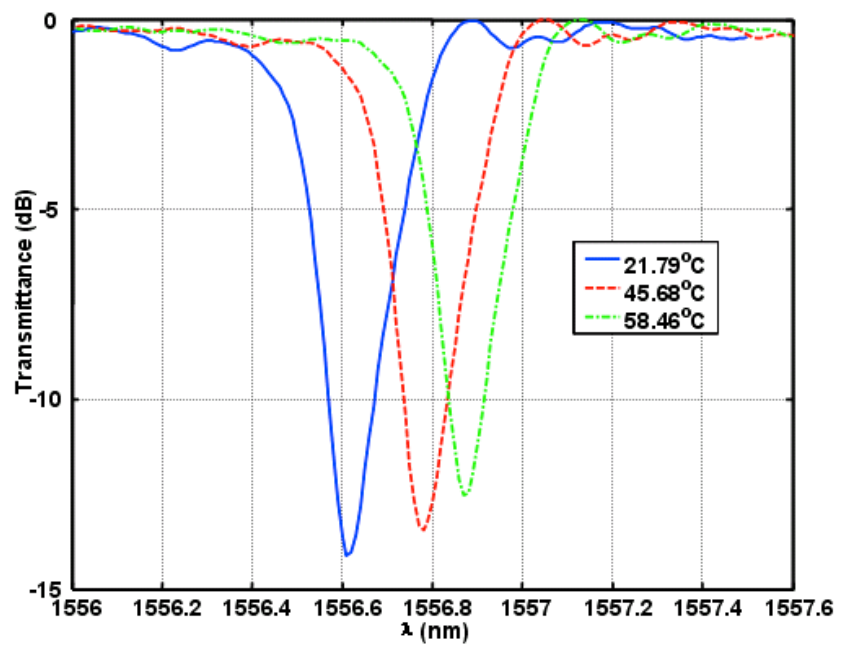

(a)

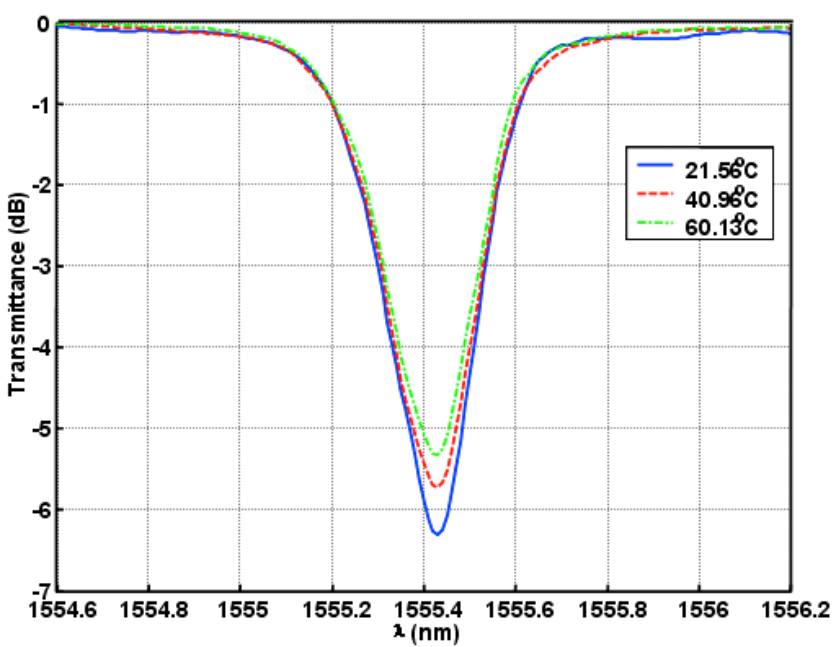

(b)

Figure 3. (a) Filter response measured in the through channel at $\theta=2.25^{\circ}$ for three different temperatures. (b) Filter response measured in the through channel at $\theta=2.25^{\circ}$ for three different temperatures with the compensating MEMS mirror.

\section{MULTI-LINE HOLOGRAPHIC FILTERS}

It is well known that within the dynamic range of the recording medium, multiple holograms can be superimposed in the same volume, and their strengths, linewidths, and spectral positions can be controlled by the recording geometry, recording medium and exposure time. This makes it possible to construct a holographic filter whose spectral response is matched precisely to the absorption spectrum of a given substance. The most common multiplexing scheme is the angle multiplexing, which is to record multiple holograms by changing the angle between the two recording beams in sequence. For example, if the angle between the recording beams is $90^{\circ}$, changing this angle by $0.03^{0}$ will shift the working wavelength by $0.4 \mathrm{~nm}$.

The multiplexing of holograms in one location causes the diffraction efficiency of each hologram to drop inversely proportional to the square of the number of holograms. Specifically, the diffraction efficiency of $M$ equal-strength holograms is ${ }^{9} \eta=(M / \# / M)^{2}$. The quantity $M / \#$ characterizes the dynamic range performance of a holographic system, 
and depends highly on the material properties and the optical system. The $1 / M^{2}$ dependence makes it necessary for us to optimize the material and system design in order to get high enough diffraction efficiency for each hologram.

The desired spectral response is written into the crystal by breaking it up into a sequence of peaks of varying amplitude and width and then recording a separate hologram for each of the peaks. The spectral center of each hologram is determined by the angle between the two recording beams. The strength of each hologram is controlled by the exposure time during hologram formation. Finally, the spectral bandwidth of each hologram is determined by the effective hologram thickness. A filter constructed in this way has a spectral response with multiple peaks at specified positions and with specified strengths and widths. To characterize the filter, a tunable near-IR laser is used to illuminate the filter from one side (reflection geometry). Then the magnitude spectral response of the filter is obtained by measuring the transmission efficiency.

After a hologram is recorded in the medium, any successive recording will cause an incoherent erasure to this hologram. Thus a specially designed exposure schedule must be applied to ensure the required relative strength of each hologram, and to fully utilize the dynamic range of the material. An exposure schedule for multiplexing equal strength holograms in photopolymer materials was introduced by Allen $\mathrm{Pu}$ et. al. ${ }^{10}$. The same principle can be applied here to record multiple unequal strength holograms in a photorefractive crystal. First, the recording curve of the material, which is the cumulative hologram strength variation curve as a function of exposure energy, is measured and the dynamic range of the material $(\mathrm{M} / \#)$ is determined. Then the exposure time for each hologram is calculated by allocating the dynamic range unequally according to the required strength of each hologram.

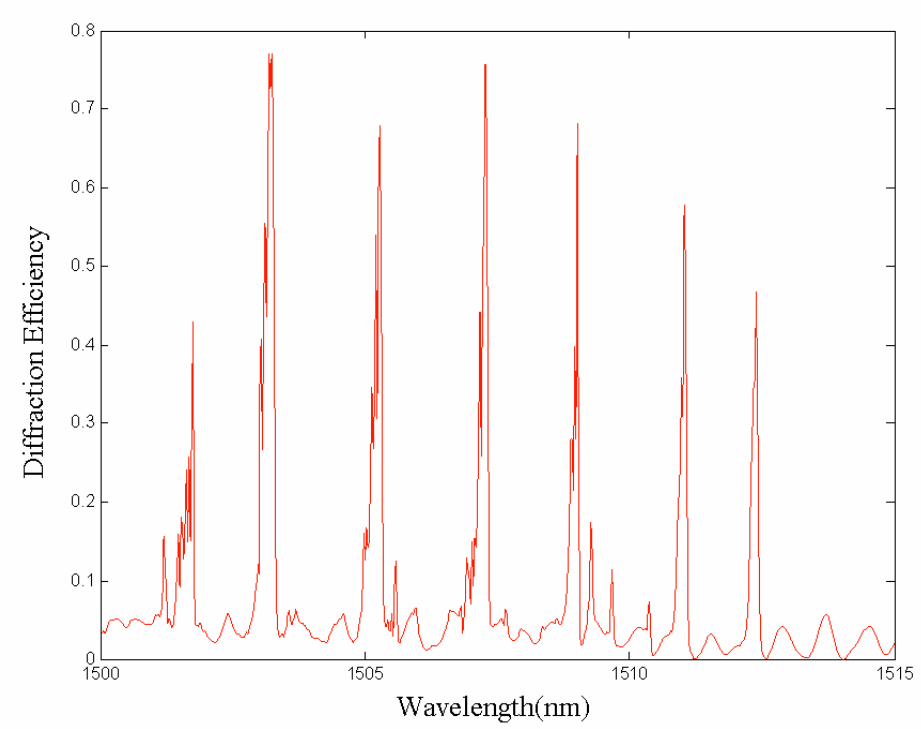

Figure 4. Measured magnitude spectral response of the multi-line holographic filter recorded using angle-multiplexing method.

A volume holographic filter with seven holograms multiplexed in the same volume is recorded as described above. The spectral response of the filter is shown in Fig. 4. The recording angle (angle between the recording beams) for the first peak in the spectrum from the left is $90^{\circ}$ outside the crystal, and each time $0.03^{\circ}$ is subtracted for the following peak. The recording schedule is $75 \mathrm{~min}$ (for the first hologram) $->60 \mathrm{~min}->50 \mathrm{~min}->40 \mathrm{~min}->30 \mathrm{~min}->20 \mathrm{~min}->10 \mathrm{~min}$. The average diffraction efficiency of the resulting holograms is $\sim 60 \%$. The linewidth (FWHM) of each peak is less than $0.1 \mathrm{~nm}$. The sidelobes on the left side of each peak is caused by a nonzero DC refractive index modulation. Such a multiline holographic filter has the potential to greatly increase the sensitivity of spectroscopic gas measurement while decreasing the volume of data generated.

Future work is aimed at optimizing the recording schedule to record more holograms and better control their relative strengths. Then we plan to apply this type of filter to both spectroscopic and microscopic applications. For spectroscopy, we will use this filter to enhance the sensitivity of traditional grating spectrometers by integrating multiple spectral lines. For microscopy, we will use it to image different spectral components of the sample, which is very useful for fluorescent imaging systems. 


\section{CONCLUSION}

Two novel realizations of volume holographic filters have been presented and demonstrated experimentally. An athermal design combining VHG and a passive MEMS mirror keeps the center wavelength of the filter constant from $21^{\circ} \mathrm{C}$ to $60^{\circ} \mathrm{C}$. In practice, the packaging of such a device should be such that the mirror and the volume grating thermally track each other. A multi-line holographic filter is demonstrated by multiplexing seven holograms in the same $\mathrm{LiNbO}_{3}: \mathrm{Fe}$ crystal. The average diffraction efficiency of the holograms is $60 \%$. The linewidth (FWHM) of each hologram is less than $0.1 \mathrm{~nm}$. Such filters can be used to emulate the $\mathrm{CO}_{2}$ absorption spectrum in the 1.6um band and greatly increase the spectroscopic measurement of the atmospheric $\mathrm{CO}_{2}$ concentration.

\section{ACKNOWLEDGEMENT}

This work was funded by the NSF Engineering Research Center on Neuromorphic Systems Engineering.

\section{REFERENCES}

1. G. C. Valley, M.B. Klein, R. A. Mullen, D. Rytz, B. Wechsler, "Photorefractive materials", Annual Review of Materials Science 18: 165-188 1988.

2. J. E. Ludman, J. R. Riccobono, N. O. Reinhand, I. V. Semenova, Y. L. Korzinin, S. M. Shahriar, H. J. Caulfield, J. M. Fournier, P. Hemmer, "Very thick holographic nonspatial filtering of laser beams", Optical Engineering 36(6): 1700-1705 JUN 1997.

3. A. Yariv, P. Yeh, Optical Waves in Crystals, John Wiley \& Sons, Inc., New York, 1984.

4. Y. Inoue, A. Kaneko, F. Hanawa, H. Takahashi, K. Hattori, S. Sumida, "Athermal silica-based arrayed-waveguide grating multiplexer", S Electronic Letters 33 (23): 1945-1947 NOV 61997.

5. S. Yoshihara, T. Matano, H. Ooshima, A. Sakamoto, "Reliability of athermal fiber Bragg grating component with negative thermal expansion ceramic substrate", IEICE Transactions on Electronics E86C (12): 2501-2503 DEC 2003.

6. R. T. Smith, F. S. Welsh, "Temperature dependence of elastic, piezoelectric, and dielectric constants of lithium tantalite and lithium niobate," Journal of Applied Optics, vol. 42, 2219-2230, MAY 1971.

7. U. Schlarb and K. Betzler, "Refractive indices of lithium niobate as a function of temperature, wavelength, and composition - a generalized fit," Physical Review B, vol. 48, 15613-15620, DEC 1993.

8. D. L. Staebler and J. J. Amodei, "Thermally fixed holograms in $\mathrm{LiNbO}_{3}$," Ferroelectrics, vol. 3, pp. 107-113, 1972.

9. F.H. Mok, G.W. Burr, and D. Psaltis, "System metric for holographic memory systems", Opt. Lett. 21 (12), 896898, JUN 151996.

10. A. Pu, K. Curtis, and D. Psaltis, "Exposure schedule for multiplexing holograms in photopolymer films", Opt. Eng. 35 (10), 2824-2829, OCT 1996. 